\title{
LIMIT CYCLES \\ FOR TWO FAMILIES OF CUBIC SYSTEMS
}

\author{
M.J. ÁlVAREZ, A. GASUlL, AND R. PROHENS
}

\begin{abstract}
In this paper we study the number of limit cycles of two families of cubic systems introduced in previous papers to model real phenomena. The first one is motivated by a model of star formation histories in giant spiral galaxies and the second one comes from a model of Volterra type. To prove our results we develop a new criterion on non-existence of periodic orbits and we extend a well-known criterion on uniqueness of limit cycles due to Kuang and Freedman. Both results allow to reduce the problem to the control of the sign of certain functions that are treated by algebraic tools. Moreover, in both cases, we prove that when the limit cycles exist they are non-algebraic.
\end{abstract}

\section{INTRODUCTION}

In this work we study the number of limit cycles of two 2-parameter families of planar cubic systems introduced in previous papers to model real phenomena. The first one is the planar system

$$
\left\{\begin{array}{l}
\dot{x}=A(1-x-y)-B x y^{2} \\
\dot{y}=-y(1-x-y)+B x y^{2}
\end{array}\right.
$$

where $A$ and $B$ are positive real parameters. It turns out to be one of the simplest models for the formation of spiral galaxies. In Section 4 we briefly summarize how to derive it, following the explanation of [11]. The study developed in [11] is mainly numeric. In that work, it is proved that a limit cycle appears, via an Andronov-Hopf bifurcation, when crossing the curve $B=(1-2 A) / A^{2}$ and it always exists for $B<(1-2 A) / A^{2}$. Moreover some numerical evidences are presented to illustrate the situation. Here we make a complete analytic study of the system, characterizing the existence and uniqueness of the limit cycle. We prove:

Theorem A. Consider system (1.1) in the first quadrant with $A>0, B>0$. It has a periodic orbit if and only if $B<(1-2 A) / A^{2}$. Moreover in this case it is unique, stable and non-algebraic.

The second system is a predator-prey one of Volterra type that in dimensionless variables writes as

$$
\left\{\begin{array}{l}
\dot{x}=x(x(1-x)-(x+n) y) \\
\dot{y}=y(x+n)(x-m)
\end{array}\right.
$$

2000 Mathematics Subject Classification. Primary: 34C07. Secondary: 37C10, 37N20, $37 \mathrm{~N} 25$.

Key words and phrases. Cubic system, Kolmogorov system, limit cycle, bifurcation.

The first and second authors are supported by grant MTM2008-03437 and the second author also by 2009SGR 410 . 
with $n$ and $m$ positive parameters. See Section 5 for a short motivation for the study of this system, following [3]. In that paper it is proved that there are parameters near the curve $m=\sqrt{n^{2}+n}-n$ where one or two limit cycles appear via codimension one and two Andronov-Hopf bifurcations. In particular the codimension two bifurcation takes place at the point $(n, m)=$ $(1 / 8,1 / 4)=: H_{2}$. We prove:

Theorem B. Consider system (1.2) and define the following regions:

$$
\begin{aligned}
& R_{0}=\left\{(n, m): n>0, m \geq \max \left(\sqrt{n^{2}+n}-n, \frac{(2 n+1)^{2}}{4 n^{2}+8 n+5}\right)\right\}, \\
& R_{1}=\left\{(n, m): n>0, m<\min \left(\sqrt{n^{2}+n}-n, \frac{2 n}{1+3 n}\right)\right\}, \\
& R_{2}=\{(n, m): m>0, n>0\} \backslash\left(R_{0} \cup R_{1}\right),
\end{aligned}
$$

see Figure 1. Then the following holds:

(i) For $(n, m) \in R_{0}$ it does not have periodic orbits.

(ii) For $(n, m) \in R_{1}$ it has exactly one periodic orbit, which is a stable limit cycle.

(iii) There are values of $(n, m) \in R_{2}$ such that the system has at least two limit cycles with opposite stabilities. In particular, this happens when $n \in(0,1 / 8)$ and $m \gtrsim \sqrt{n^{2}+n}-n$.

Moreover, in all cases, the existing limit cycles are non-algebraic.

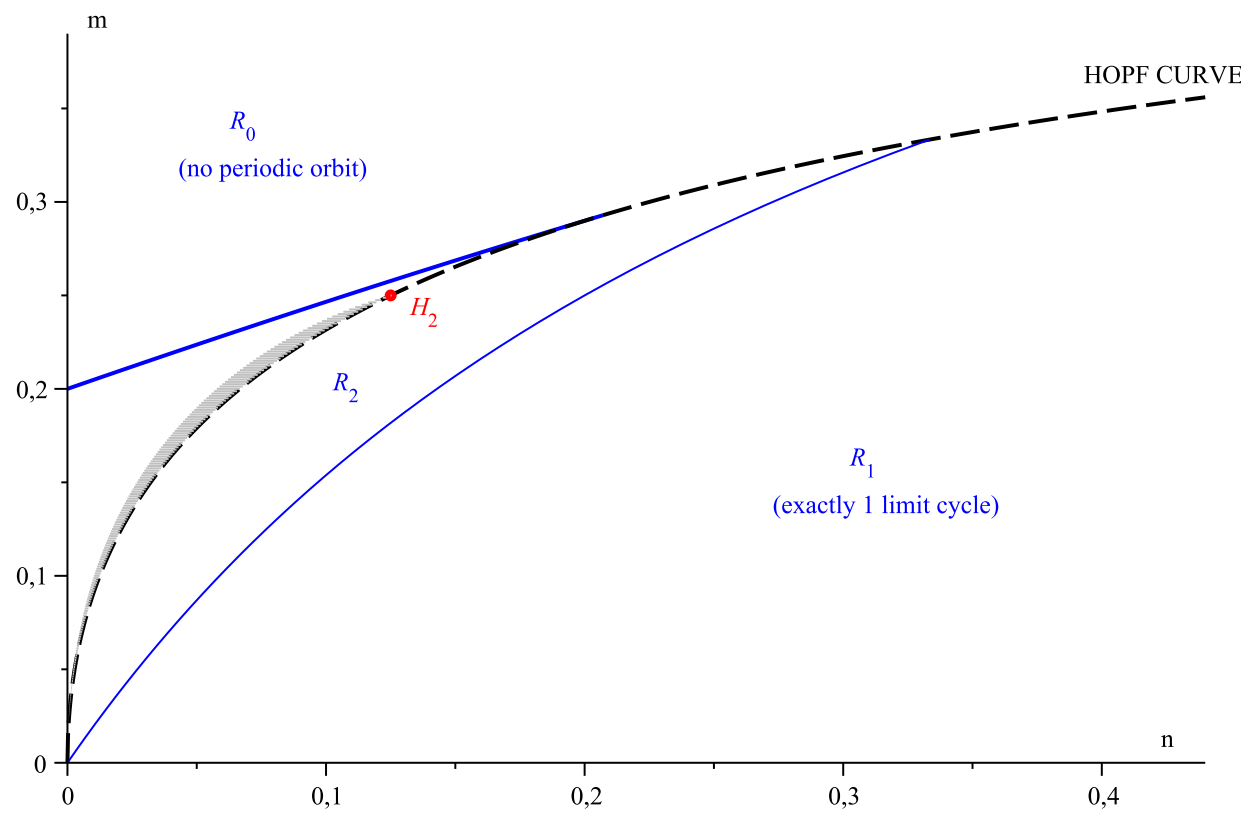

Figure 1. Bifurcation diagram for system (1.2). In the small grey region we know the existence of at least two limit cycles. 
Remark 1.1. In [3] the authors conjecture that the maximum number of limit cycles of system (1.2) is two and its bifurcation diagram is the one given in Figure 2. In particular they assert that on a curve above the Hopf curve, starting at $(0,0)$ and ending at $H_{2}$, there is a blue-sky bifurcation and on it the system has a unique semistable limit cycle. In Figure 2 this curve is qualitative plotted by a solid line. Notice that our theorem proves the conjectured bifurcation diagram on regions $R_{0}$ and $R_{1}$. Moreover our results on the region $R_{2}$ are also coherent with the ones showed in Figure 2 and in particular with the existence of the blue-sky bifurcation, see also Proposition 5.1(v).

We have not performed an exhaustive numerical study, but all our computations are also coherent with their conjecture. For instance when $m=0.15$ we have obtained that the two limit cycles seem to disappear for a value $\widetilde{n}=0.02045 \pm 5 \times 10^{-5}$.



Figure 2. The numbers appearing in the figure denote the number of limit cycles for system (1.2) conjectured in [3].

The proofs of both theorems follow similar steps. We study the nonexistence of limit cycles by using a new criterion given in Subsection 2.1, see Theorem 2.1. The proof of this result uses the Bendixson-Dulac Criterion and extends some previous results of [14]. The regions of uniqueness of the limit cycle are given by using an extension of a result of Kuang and Freedman [15] that will be given in Subsection 2.2. Finally the non-algebraicity of the existing limit cycles is proved by applying a method introduced in [5], briefly recalled in Subsection 2.3.

The paper is organized as follows: Section 2 is devoted to present all the preliminary results. In Section 3, as a first illustration of the tools used in this paper, we present a complete study of the known results for the model of Rosenzweig and McArthur. In this case, as we will see, by using our approach the proofs of non-existence and uniqueness of periodic orbits are really simple. Section 4 is devoted to prove Theorem A and Section 5 to prove Theorem B. 


\section{Preliminary Results.}

2.1. New criterion on the non-existence of limit cycles. In this subsection we give a criterion of non-existence of limit cycles for a family of Kolmogorov systems. We prove:

Theorem 2.1. Consider system

$$
\mathcal{X}(x, y)=\left\{\begin{array}{l}
\dot{x}=x\left(g_{0}(x)+g_{1}(x) y+g_{2}(x) y^{2}\right), \\
\dot{y}=y\left(h_{0}(x)+h_{1}(x) y+h_{2}(x) y^{2}\right),
\end{array}\right.
$$

defined for all $x \geq 0, y \geq 0$ and where all the functions are of class $\mathcal{C}^{1}$. For any $\lambda \in \mathbb{R}$ define the functions:

$S_{\lambda}(x)=x\left[g_{0}^{\prime}(x) g_{1}(x)-g_{0}(x) g_{1}^{\prime}(x)\right]+\lambda h_{0}(x) g_{1}(x)-(1+\lambda) g_{0}(x) h_{1}(x)$,

$T_{\lambda}(x)=x\left[g_{2}^{\prime}(x) g_{1}(x)-g_{2}(x) g_{1}^{\prime}(x)\right]+(2+\lambda) h_{2}(x) g_{1}(x)-(1+\lambda) g_{2}(x) h_{1}(x)$.

Let $I \subset \mathbb{R}^{+}$be an open interval. We also introduce the following assumptions:

(i) Either $g_{1}(x) \neq 0$, for all $x \in I$, or $g_{2} \equiv 0$ and, in any case, there exists a value of $\lambda$ such that $S_{\lambda}(x) T_{\lambda}(x) \geq 0$, for all $x \in I$, and all zeroes of $S_{\lambda}(x) T_{\lambda}(x)$ are isolated.

(ii) $h_{2} \equiv g_{2} \equiv 0$ and there exists a $\lambda$ such that $S_{\lambda}(x)$ does not change sign, for all $x \in I$, and all its zeroes are isolated.

If one of the assumptions (i) or (ii) holds, then system (2.1) does not have periodic orbits totally included in the strip $I \times(0,+\infty)$.

Moreover, if there exists a $\lambda$ such that $S_{\lambda} \equiv T_{\lambda} \equiv 0$, then system (2.1) is integrable.

Proof. First, let us assume that $g_{2} \equiv 0$. In this case, if there exists a limit cycle then it can not intersect the set $\left\{(x, y) \mid x>0, g_{1}(x)=0\right\}$. This holds because if $\bar{x}>0$ is such that $g_{1}(\bar{x})=0$ then either $x=\bar{x}$ is an invariant line (i.e. $g_{0}(\bar{x})=0$ ) or it is a line without contact, i.e $\left.\dot{x}\right|_{x=\bar{x}}=\bar{x} g_{0}(\bar{x}) \neq 0$. Hence, in the region where our system can have periodic orbits we can always assume that $g_{1}$ does not vanish.

To prove the results we look for a suitable Dulac function of the form $B(x, y)=y^{\lambda-1} Z(x)$. Simple computations give that

$$
\begin{aligned}
D(x, y):= & \operatorname{div}(B(x, y) \mathcal{X}(x, y))= \\
& {\left[\left(x g_{0}(x) Z(x)\right)^{\prime}+\lambda h_{0}(x) Z(x)\right.} \\
+ & \left(\left(x g_{1}(x) Z(x)\right)^{\prime}+(\lambda+1) h_{1}(x) Z(x)\right) y \\
+ & \left.\left(\left(x g_{2}(x) Z(x)\right)^{\prime}+(\lambda+2) h_{2}(x) Z(x)\right) y^{2}\right] y^{\lambda-1} .
\end{aligned}
$$

The solutions of the differential equation

$$
\left(x g_{1}(x) Z(x)\right)^{\prime}+(\lambda+1) h_{1}(x) Z(x)=0
$$

are

$$
Z_{x_{0}}(x)=\frac{\exp \left[-(\lambda+1) \int_{x_{0}}^{x} \frac{h_{1}(s)}{s g_{1}(s)} d s\right]}{x g_{1}(x)}
$$


varying $x_{0}$. Recall that these functions, for $x_{0}>0$, are well defined in the regions where the periodic orbits can lie, because $g_{1}$ does not vanish on them.

By taking the Dulac function $\tilde{B}(x, y)=y^{\lambda-1} Z_{x_{0}}(x)$, for a given $x_{0}>0$, and by using that $Z_{x_{0}}(x)$ satisfies the differential equation (2.2), after doing some computations, we get

$$
D(x, y)=\frac{Z_{x_{0}}(x)}{g_{1}(x)}\left(S_{\lambda}(x)+T_{\lambda}(x) y^{2}\right) y^{\lambda-1} .
$$

Let us take a value of $\lambda$ for which $S_{\lambda}(x) T_{\lambda}(x) \geq 0$ in $I$. We have that the function $D(x, y)$ does not change sign in $I \times(0,+\infty)$ and vanishes on a set of zero Lebesgue measure. Hence, by the Dulac Criterion, the result is proved.

In case (ii), note that

$$
D(x, y)=\frac{Z_{x_{0}}(x) S_{\lambda}(x) y^{\lambda-1}}{g_{1}(x)}
$$

and the result follows similarly.

Finally notice that when $S_{\lambda}(x) \equiv T_{\lambda}(x) \equiv 0$ the function $B(x, y)$ is an integrating factor of the system.

Observe that the function $S_{\lambda}(x)$ of Theorem 2.1 can also be written as

$$
S_{\lambda}(x)=g_{1}^{2}(x)\left[x\left(\frac{g_{0}(x)}{g_{1}(x)}\right)^{\prime}+\lambda \frac{h_{0}(x)}{g_{1}(x)}-(1+\lambda) \frac{g_{0}(x) h_{1}(x)}{g_{1}^{2}(x)}\right] .
$$

When $h_{1}(x) \equiv 0$, it essentially coincides with the one given in the nonexistence criterion presented in [14, Thm 4.1]. Of course, $T_{\lambda}(x)$ can be also written in a similar way.

Note that our results on system (2.1) can be applied to several Kolmogorov type systems. For instance they perfectly match with the Gausetype systems considered in [17], which have the form

$$
\left\{\begin{array}{l}
\dot{x}=x g(x)-y p(x), \\
\dot{y}=\gamma y\left(-\delta+h(x)-\eta y-\alpha y^{2}\right),
\end{array}\right.
$$

or with the system

$$
\left\{\begin{array}{l}
\dot{x}=x\left(a_{1}+a_{2} x-a_{3} x^{2}-k y\right) \\
\dot{y}=y(-1+x+y)
\end{array}\right.
$$

studied in [10]. See also the systems considered in [9].

In this paper we will apply our criterion to prove the non-existence results in Theorems A and B, but as a first illustration we will use it to prove that under some hypotheses a simple Kolmogorov system does not have limit cycles. We prove:

Proposition 2.2. The system,

$$
\left\{\begin{array}{l}
\dot{x}=x\left(a+b x+c y+d y^{2}\right) \\
\dot{y}=y\left(e+f x+g y+h y^{2}\right)
\end{array}\right.
$$

where all the parameters are real numbers satisfying 
(i) either $c f-b g \neq 0$ and

$$
(a b g+b e g-c b e-a f g)(2 c f h+b d g-b g h-c b h-d f g)>0 ;
$$

(ii) or $d=h=0$,

does not have limit cycles in the first quadrant.

We remark that in the above proposition we fix our attention into the first quadrant but similar results can be obtained for the whole plane. Notice also that item (ii) proves in particular the well-known fact that the classical competitive Lotka-Volterra model in two dimensions does not have limit cycles for any value of the parameters. Recall that it can be written as

$$
\left\{\begin{array}{l}
\dot{x}=r_{1} x\left(1-\frac{x}{K_{1}}-\frac{\alpha_{12}}{K_{1}} y\right), \\
\dot{y}=r_{2} y\left(1-\frac{y}{K_{2}}-\frac{\alpha_{21}}{K_{2}} x\right),
\end{array}\right.
$$

where all the appearing parameters are positive.

Proof of Proposition 2.2. In order to apply Theorem 2.1 notice that:

$$
\begin{aligned}
& g_{0}(x)=a+b x, \quad g_{1}(x)=c, \quad g_{2}(x)=d, \\
& h_{0}(x)=e+f x, \quad h_{1}(x)=g, \quad h_{2}(x)=h,
\end{aligned}
$$

and we take $I=(0, \infty)$. Straightforward computations give that

$$
\begin{aligned}
& S_{\lambda}(x)=[(c f-b g) \lambda+b(c-g)] x+[(c e-a g) \lambda-a g], \\
& T_{\lambda}(x)=(c h-d g) \lambda+2 c h-d g,
\end{aligned}
$$

(i) Since we assume that $c f-b g \neq 0$ we can take $\lambda=\lambda^{*}:=b(g-c) /(c f-$ $b g$ ) and then

$$
\begin{aligned}
& S_{\lambda^{*}}(x)=\frac{c(a b g+b e g-c b e-a f g)}{c f-b g}, \\
& T_{\lambda^{*}}(x)=\frac{c(2 c f h+b d g-b g h-c b h-d f g)}{c f-b g} .
\end{aligned}
$$

When $c=0$, the system is integrable and, hence, looking at its integrating factor we conclude that it does not have any limit cycle. When $c \neq 0$ we have by hypothesis that

$$
S_{\lambda}(x) T_{\lambda}(x)>0 .
$$

Therefore, by using (i) of Theorem 2.1 we obtain that it has no periodic orbits.

(ii) When $d=h=0$ and $c f-b g=0$ then either system (2.3) does not have critical points outside the invariant axes or it has a line full of critical points, but in any case it trivially does not have limit cycles. So we can assume that $c f-b g \neq 0$ and take again $\lambda=\lambda^{*}$. Then $S_{\lambda^{*}}(x)$ coincides with the above expression and $T_{\lambda^{*}}(x) \equiv 0$. By applying item (ii) of Theorem 2.1, when the above quantity $S_{\lambda^{*}}(x)$ is not zero, we know that the system does not have periodic orbits and when it is zero we have an integrable system. In any case there are no limit cycles, as we wanted to prove. 
2.2. A criterion on the uniqueness of limit cycles. In this subsection we extend a nice criterion of Kuang and Freedman, see [15], for determining the uniqueness of the limit cycle for some systems of Kolmogorov type.

Theorem 2.3. Consider system

$$
\begin{aligned}
& \dot{x}=x g(x)-y p(x), \\
& \dot{y}=y(-\gamma+q(x)),
\end{aligned}
$$

in the first quadrant, with $\gamma>0$. Assume that there exist $K>0$ and $x^{*}>0$ such that:

(i) $p(0)=0, p^{\prime}(x)>0$, for $0<x<K$,

(ii) $g(K)=0,(x-K) g(x)<0$, for $0<x \neq K$,

(iii) $q\left(x^{*}\right)=\gamma,(q(x)-\gamma)\left(x-x^{*}\right)>0$, for $x \in[0, K] \backslash\left\{x^{*}\right\}$,

(iv) the unique equilibrium point $\left(x^{*}, y^{*}\right)$ in the region $R=\{(x, y): x \in$ $[0, K], y>0\}$ is unstable,

(v) for $0<x<K$,

$$
\Phi(x):=\frac{d}{d x}\left(\frac{x g^{\prime}(x)+g(x)-x g(x) p^{\prime}(x) / p(x)}{-\gamma+q(x)}\right) \leq 0, \quad x \neq x^{*} .
$$

Then system (2.4) has exactly one periodic orbit which is a globally asymptotically stable limit cycle.

Sketch of the proof. The proof follows the same steps as the one of Kuang and Freedman's theorem, [15], and there are only two differences. The first one is that in that paper it is assumed that $g(0)>0$ to make system (2.4) as a consistent predator-prey model.

The second one is that it is assumed that $q(0)>0$ and $q^{\prime}(x)>0$, for $x>0$, to ensure that $-\gamma+q(x)$ has only one simple positive solution.

Looking carefully to the Kuang and Freedman's proof one realizes that the first condition is used nowhere while the second one is only needed to know that the system has a unique equilibrium point in the region $R$.

Remark 2.4. (i) As we will see when we apply the previous theorem to system (1.2) the fact that we have been able to remove the hypothesis that $q^{\prime}(x)>0$ increases the region of parameters for which we can prove the existence of exactly one limit cycle.

(ii) By using the well-known Rosenzweig and McArthur criterion [4, §4] we know that the hyperbolic instability of $\left(x^{*}, y^{*}\right)$ is equivalent to

$$
\left.\frac{d}{d x}\left(\frac{x g(x)}{p(x)}\right)\right|_{x=x^{*}}>0 \text {. }
$$

2.3. A method for studying the existence of algebraic solutions. In this section we recall a method developed in [5] for studying the existence of algebraic invariant curves of planar polynomial systems for which we know a concrete solution. As we will see this method works especially well for Kolmogorov systems for which the two axes are known to be invariant curves.

It is said that $F(x, y)=0$ is an algebraic solution of a planar polynomial differential equation

$$
\left\{\begin{array}{l}
\dot{x}=P(x, y) \\
\dot{y}=Q(x, y)
\end{array}\right.
$$


if $F(x, y)$ is a polynomial for which there exists another polynomial $K(x, y)$ (called the cofactor of $F$ ) such that

$$
\frac{\partial F(x, y)}{\partial x} P(x, y)+\frac{\partial F(x, y)}{\partial y} Q(x, y)=K(x, y) F(x, y) .
$$

It is clear that the set $\mathcal{F}=\left\{(x, y) \in \mathbb{R}^{2}: F(x, y)=0\right\}$ is an invariant set for the flow associated to (2.5). Notice also that the degree of $K(x, y)$ is at most one degree less that $\max (\operatorname{deg}(P(x, y)), \operatorname{deg}(Q(x, y)))$.

Fixed an analytic solution of (2.5) of the form $y=\alpha(x)$, which is not contained in the set $\mathcal{F}$, we can consider the following Taylor expansion in $z$,

$$
\Phi(x, \alpha(x)+z)=\Phi_{0}(x)+\Phi_{1}(x) z+\Phi_{2}(x) z^{2}+\ldots,
$$

where $\Phi$ is taken as one of the functions $P, Q, F$ or $K$. Notice that $\alpha^{\prime}(x)=$ $Q_{0}(x) / P_{0}(x)$. Then equation (2.6) can be written as

$$
\begin{aligned}
\sum_{k=0}^{\infty} & \left(\sum _ { i = 0 } ^ { k } \left(P_{k-i}(x) F_{i}^{\prime}(x)\right.\right. \\
& \left.\left.+\left(i Q_{k-i+1}(x)-i \alpha^{\prime}(x) P_{k-i+1}(x)-K_{k-i}(x)\right) F_{i}(x)\right)\right) z^{k}=0 .
\end{aligned}
$$

The functions $F_{k}(x)$ can be computed recurrently from the above relation by solving the linear differential equations in $F_{k}(x)$, obtained vanishing each coefficient in $z^{k}$. For instance, for $k=0$,

$$
P_{0}(x) F_{0}^{\prime}(x)-K_{0}(x) F_{0}(x)=0,
$$

and we obtain $F_{0}(x)=C_{0} \exp \left(\int_{0}^{x} K_{0}(s) / P_{0}(s) d s\right)$, where $C_{0}$ is an arbitrary constant.

When $\alpha(x)$ is a polynomial, with real or complex coefficients, the linear differential equations for each $F_{k}(x)$ described in the above algorithm give us a collection of necessary conditions for the existence of an algebraic solution $F(x, y)=0$. The conditions are that, for each $k$, the functions $F_{k}(x)$ must be polynomials.

\section{A first example: the model of Rosenzweig and McArthur}

In this section we study the number of limit cycles in the first quadrant of the system of Rosenzweig and McArthur, see [18]:

$$
\left\{\begin{aligned}
\dot{x} & =r x\left(1-\frac{x}{k}\right)_{m x}-\frac{m x}{A+x} y, \\
\dot{y} & =-\delta y+\gamma \frac{m}{A+x} y
\end{aligned}\right.
$$

with all the parameters positive. The results that we will give are not new, but we give a shorter prove that also serves to illustrate the methods that we will use along this paper. It is worth to comment that the tools that we will use, namely Theorems 2.1 and 2.3, are extensions of results proved to study the above system.

To study system (3.1) in the first quadrant it is convenient first to reparameterize the time, multiplying the vector field by the positive function $A+x$ and second to prepare a dimensionless model with the new variables 
$x_{1}=x / k, y_{1}=m y /(\delta A)$ and $t_{1}=t /(\delta A)$. After removing the subindexes we obtain system

$$
\left\{\begin{array}{l}
\dot{x}=x((1-x)(P+Q x)-y) \\
\dot{y}=y(-1+R x)
\end{array}\right.
$$

where $P>0, Q>0$ and $R \in \mathbb{R}$. In fact $P=r / \delta, Q=r k /(\delta A)$ and $R=k(m \gamma-\delta) /(\delta A)$. We prove

Theorem 3.1. Consider system (3.2) in the first quadrant with $P>0$ and $Q>0$. It has a periodic orbit if and only if $T:=P R-Q R+2 Q<0$. Moreover, in this case it is unique and stable.

Proof. The system (3.2) has a critical point in the open first quadrant only when $R>0$ and $(R-1)(P R+Q)>0$. In this case it is easy to see that it has index +1 and that the divergence of the associated vector field at this critical point is $-T / R^{2}$. Hence when $T>0$ (resp. $T<0$ ) the point is a local attractor (resp. repellor). Some further work when $T=0$, by computing the Lyapunov constant at the point, gives that in this case it is also an attracting weak focus.

To prove the existence and uniqueness of the limit cycle when $T<0$, we apply Theorem 2.3. The functions appearing in its statement are

$$
g(x)=(1-x)(P+Q x), \quad p(x)=-x, \quad q(x)=R x,
$$

$\gamma=1$ and $K=1$. Then

$$
\Phi(x)=\frac{-2 Q R x^{2}+4 Q x+P-Q}{(R x-1)^{2}} .
$$

Note that

$$
\operatorname{dis}\left(-2 Q R x^{2}+4 Q x+P-Q, x\right)=8 Q T<0,
$$

where $\operatorname{dis}(p(x), x)$ denotes the discriminant of $p(x)$ with respect to $x$. Therefore we have that $\Phi(x) \leq 0$ for $x \neq 1 / R$ and the existence and uniqueness of the limit cycle follows.

To prove the non-existence of periodic orbits when $T \geq 0$ we use item (ii) of Theorem 2.1 by taking

$$
g_{0}(x)=(1-x)(P+Q x), \quad g_{1}(x)=-1, \quad h_{0}(x)=-1+R x, \quad h_{1}(x) \equiv 0 .
$$

Then

$$
S_{\lambda}(x)=2 Q x^{2}+(P-Q-\lambda R) x+\lambda .
$$

Observe that if we find $\lambda$ such that $S_{\lambda}$ has a double root we are done. Note that

$$
d(\lambda):=\operatorname{dis}\left(S_{\lambda}(x), x\right)=\lambda^{2}+(2 R(Q-P)-8 Q) \lambda+(P-Q)^{2}
$$

and since

$$
\operatorname{dis}(d(\lambda), \lambda)=3 Q T \geq 0,
$$

such a $\lambda$ always exists and the non-existence of periodic orbits follows.

Remark 3.2. Notice that in the original parameters of the Rosenzweig and McArthur model

$$
T=\frac{k r}{(\delta A)^{2}}(\delta(A+k)+\gamma m(A-k)) .
$$




\section{A SYSTEM MODELING THE CREATION OF SPIRAL GALAXIES}

4.1. Deduction of the model. As we have already said, in this subsection we briefly recall the explanation of [11] for the deduction of system (1.1). For more details, see this reference.

We concentrate in a scenario of star formation histories in giant spiral galaxies. By observations of soft X-ray background and O VI absorption lines, since the late 70's it is admitted that the interstellar medium consists of three components, instead of the two considered until then. These three components are warm gas, cold clouds and, the new one, hot gas. Their relative abundances are denoted by $X_{w}, X_{c}, X_{h}$, respectively. The interchange processes among these three components are regulated by supernova remnants through the following three interactions:

- the sweeping of a warm gas into a cold component, that can be approximated by $a X_{w}$. Although the coefficient $a$ depends on the variables, in this model is set to be constant.

- the evaporation of cold clouds embedded in a hot gas, that is proportional to the abundance of clouds and the temperature of the hot gas component in which clouds are embedded. This factor can be taken as $b X_{c} X_{h}^{2}$. As in the previous item, although the complexity of the function $b$, it is not very restrictive to take it as a constant.

- the radiative cooling of a hot gas by collisions with a warm gas, that it is assumed to be proportional to the collision rate between the hot and the warm gases, as $c X_{w} X_{h}$.

For all the previous considerations, the rate equations for the time variation of each component are

$$
\left\{\begin{array}{l}
\frac{d X_{w}}{d \tau}=-a X_{w}+c X_{h} X_{w}, \\
\frac{d X_{c}}{d \tau}=-b X_{c} X_{h}^{2}+a X_{w}, \\
\frac{d X_{h}}{d \tau}=-c X_{h} X_{w}+b X_{c} X_{h}^{2} .
\end{array}\right.
$$

Since the three variables are related by $X_{w}+X_{c}+X_{h}=1$, the previous system can be reduced to system (1.1) by denoting $X_{c}=x, X_{h}=y, A=$ $a / c, B=b / c$ and doing the rescaling $t=c \tau$.

This system turned out to be important to describe the cyclical formation of galaxies and several interpretations have been given to this periodic behavior, see $[7,8,13]$, and a slight modification of it to apply it to dwarf galaxies at [12].

4.2. Non-existence and uniqueness of the limit cycle. We split the proof of Theorem A in several steps. The non-algebraicity of the existing limit cycles will be proved in next subsection.

Lemma 4.1. Consider system (1.1) with $A>0$ and $B>0$.

(i) When $B<(1-2 A) / A^{2}$ then it has at least one periodic orbit in the first quadrant.

(ii) When $A \geq 1$ it does not have periodic orbits in the first quadrant. 
Proof. The system has the critical points $(1,0),(0,1)$ and $p:=\left(\frac{1-A}{1+A B}, A\right)$. Notice that $p$ is in the open first quadrant only when $A<1$, so item (ii) holds. From now one we will assume that this inequality is satisfied.

It is easy to see that the point $(0,1)$ is an hyperbolic saddle and, by using the results given in [1], that $(1,0)$ is a semi-hyperbolic saddle-node. Moreover the determinant of the differential of the vector field associated to system (1.1) at $p$ is $(1-A) A^{2} B>0$ and its trace $A B\left(1-A^{2} B-2 A\right) /(1+A B)$. When the trace vanishes, that is when $B=(2 A-1) / A^{2}$, we compute the first Lyapunov quantity by using $[2,6]$, obtaining

$$
V_{3}=\frac{\pi}{4 A^{2}(A-1)} \sqrt{\frac{1-2 A}{1-A}} .
$$

Notice that $V_{3}<0$ and it is always well defined, because when $A \geq 1 / 2$ for no positive $B$ the trace vanishes. Hence the point $p$ is an attractor when $B \geq(2 A-1) / A^{2}$ and a repellor otherwise.

On the other hand the straight lines $y=0$ and $x+y=1$ are invariant for the flow of the system and the straight line $x=0$ is a line without contact for $0 \leq y<1$. Taking into account all the above information and by studying the location of the separatrices of the critical points $(1,0)$ and $(0,1)$ we obtain that the triangle formed by the three lines is positively invariant by the flow of the system. Since when $B<(1-2 A) / A^{2}$ we know that $p$ is an unstable focus, by using the Poincaré-Bendixson Theorem, there must exist at least one limit cycle surrounding this point, as we wanted to prove.

Next lemma converts system (1.1) into a new one suitable for a direct application of the criterion introduced in the previous section.

Lemma 4.2. System (1.1) in the first quadrant and system

$$
\left\{\begin{array}{l}
\dot{x}=x(B x(1-x)-(B x+1) y) \\
\dot{y}=y(-A+x)
\end{array}\right.
$$

also in the first quadrant, are affine conjugated.

Proof. The change of variables $x_{1}=y, y_{1}=1-x-y$, transforms one system into the other, after omitting the subindexes. Notice that this change passes the invariant lines of system (1.1) into the coordinate axes of the new system, which is of Kolmogorov type. In this new expression, the segment of the line $x+y=1$, which is in the first quadrant, is without contact.

Theorem 4.3. Consider system (4.2) and assume that $A>0, B>0$ and $B<(1-2 A) / A^{2}$. Then it has exactly one periodic orbit in the first quadrant which is a stable limit cycle.

Proof. We apply Theorem 2.3 to the system. The functions appearing in its statement are

$$
g(x)=B x(1-x), \quad p(x)=x(1+B x), \quad q(x)=x,
$$

$\gamma=A$ and $K=1$. All the hypotheses, except the stability of the equilibrium $\left(x^{*}, y^{*}\right)$ and that $\Phi(x) \leq 0$ are straightforward. By the Rosenzweig and McArthur criterion, see Remark 2.4.(ii), or by the proof of Lemma 4.1 we know that, for these values of the parameters, the equilibrium is unstable. 
Let us study the sign of the function $\Phi$. We have that

$\Phi(x)=\frac{B\left(-B^{2} x^{4}+2 B(-1+A B) x^{3}+(-2+5 A B-B) x^{2}+4 A x-A\right)}{(1+B x)^{2}(A-x)^{2}}$.

Clearly it suffices to control the sign of

$\phi_{A, B}(x):=-B^{2} x^{4}+2 B(-1+A B) x^{3}+(-2+5 A B-B) x^{2}+4 A x-A$

for $0<x<1,0<A<1 / 2$ and $B>0$.

First, notice that $\phi_{A, B}(0)=-A<0$ and $\phi_{A, B}(1)=(B+1)(2 A B+3 A-$ $B-2)<-(B+1) / 2<0$. Moreover the discriminant of $\phi_{A, B}(x)$ with respect to $x$ is

$$
\operatorname{dis}\left(\phi_{A, B}(x), x\right)=16 B^{2} A(B+1)\left(1-2 A-B A^{2}\right) \Delta(A, B)
$$

where

$\Delta(A, B):=B^{3}(27 B+28) A^{3}+3 B^{2}(20+19 B) A^{2}-3 B\left(8 B^{2}+5 B-4\right) A+(B+2)^{3}$.

We claim that, on the region $0<A<1 / 2, B>0, \Delta(A, B) \geq 0$ and $\Delta(A, B)=0$ if and only if $A=\tilde{A}:=(3-\sqrt{5}) / 8$ and $B=\tilde{B}:=8+4 \sqrt{5}$.

From the continuous dependence of the roots of a polynomial with respect to parameters we know that the number of real roots of $\phi_{A, B}(x)$ in $[0,1]$ can only vary when the above discriminant vanishes. Consider the set

$$
\mathcal{S}:=\left\{(A, B): 0<A<1 / 2,0<B<(1-2 A) / A^{2}\right\} \backslash\{(\tilde{A}, \tilde{B})\} .
$$

From the above claim, we know that on $\mathcal{S}$ the discriminant of $\phi_{A, B}(x)$ does not vanish. Since $\mathcal{S}$ is a path-connected domain, all the functions $\phi_{A, B}$, for $(A, B) \in \mathcal{S}$, have the same number of real roots in $[0,1]$. Notice that for $x \in[0,1]$,

$$
\phi_{\tilde{A}, \tilde{B}}(x)=-\frac{9+4 \sqrt{5}}{16}\left(16 x^{2}+(6 \sqrt{5}-14) x+7-3 \sqrt{5}\right)^{2} \leq 0
$$

and, for instance, $\phi_{1 / 4,4}(x)=-16 x^{4}-x^{2}+x-1 / 4<0$. Therefore we have proved that $\Phi(x) \leq 0$ and the theorem follows.

Finally, let us prove the claim. We will use similar arguments that for studying $\phi_{A, B}(x)$. Consider $\Delta_{B}(A):=\Delta(A, B)$ and think $B$ as a parameter. We have that $B^{3}(27 B+28)>0, \Delta(0, B)=(B+2)^{3}>0$ and $\Delta(1 / 2, B)=$ $\left(27 B^{4}+54 B^{3}+108 B^{2}+144 B+64\right) / 8>0$. Moreover

$$
\operatorname{dis}(\Delta(A, B), A)=-19683\left(B^{2}-16 B-16\right)^{2}(B+1)^{4} B^{6} .
$$

Hence the number of real roots can only vary when $B^{2}-16 B-16=0$, that is when $B=8+4 \sqrt{5}$. Since, for $A>0$,

$$
\begin{aligned}
\Delta(A, 0) & =8>0, \\
\Delta(A, 8+4 \sqrt{5}) & =\frac{4613+2063 \sqrt{5}}{38}(152 A-75+55 \sqrt{5})(8 A-3+\sqrt{5})^{2} \geq 0, \\
\Delta(A, 17) & =2392631 A^{3}+297381 A^{2}-122043 A+6859>0,
\end{aligned}
$$

we have proved the claim. 
With the above two results we have proved the existence, uniqueness and stability of the limit cycle when $A, B>0$ and $B<(1-2 A) / A^{2}$ for system (4.2) and equivalently for system (1.1). Let us prove the non-existence in the complementary region.

Theorem 4.4. When $A>0, B>0$ and $B \geq(1-2 A) / A^{2}$ the system (4.2) does not have periodic orbits in the first quadrant.

Proof. By Lemma 4.1 it is not restrictive to assume that $A<1$. During all the proof we will assume this inequality and the ones of the statement. We apply the criterion given in item (ii) of Theorem 2.1 by taking

$$
g_{0}(x)=B x(1-x), \quad g_{1}(x)=-1-B x, \quad h_{0}(x)=x-A, \quad h_{1}(x) \equiv 0
$$

and $I=(0,1)$, because all the periodic orbits of the system have to be in the strip $(0,1) \times(0,+\infty)$. We have that

$$
S_{\lambda}(x)=B^{2} x^{3}+(2-\lambda) B x^{2}+(\lambda A B-\lambda-B) x+\lambda A .
$$

Recall that to prove the non-existence of limit cycles it will be enough with finding a $\lambda$ such that $S_{\lambda}(x)$ does not change sign for $x \in[0,1]$.

Observe that

$$
\lim _{x \rightarrow-\infty} S_{\lambda}(x)=-\infty, \quad S_{\lambda}(0)=\lambda A .
$$

If we set $\lambda>0$ we already know that the function $S_{\lambda}(x)$ has at least one negative zero. Moreover, $S_{\lambda}(1)=(B+1)(B+\lambda A-\lambda)$. As we want $S_{\lambda}$ not to change sign for $x \in[0,1]$ then we will search $\lambda$ satisfying also that $\lambda<B /(1-A)$.

Summarizing, for each couple of given parameters $A, B$, we want to find $\lambda \in(0, B /(1-A))$ such that $S_{\lambda}(x)$ does not change sign for $x \in[0,1]$. In fact, if for each couple of parameters, we are able to find $\lambda$ in this interval such that $S_{\lambda}(x)$ has a double zero then the proof is completed, because it will not change sign.

To study the double zeroes of $S_{\lambda}$ we compute its discriminant with respect to $x$. We obtain

$$
\begin{aligned}
d_{A, B}(\lambda) & :=\operatorname{dis}\left(S_{\lambda}(x), x\right)= \\
& B^{2}\left[(1+A B)^{2} \lambda^{4}+c_{3} \lambda^{3}+c_{2} \lambda^{2}+c_{1} \lambda+4 B^{2}(B+1)\right],
\end{aligned}
$$

where $c_{1}, c_{2}$ and $c_{3}$ are given polynomials depending on $A, B$. Recall that when, for some fixed $\lambda, A$ and $B$, the polynomial $S_{\lambda}$ has a double zero then its discriminant is zero. Hence, for the given values of $A$ and $B$, we want to find $\lambda_{A, B} \in(0, B /(1-A))$ such that $d_{A, B}\left(\lambda_{A, B}\right)=0$.

Note that the function $d_{A, B}$ satisfies:

$$
\begin{gathered}
d_{A, B}(0)=4 B^{4}(1+B)>0 \\
d_{A, B}\left(\frac{B}{1-A}\right)=\frac{B^{4}\left(4-8 A+4 A^{2}+A^{2} B^{2}\right)(-2+3 A-B+2 A B)^{2}}{(1-A)^{4}}>0 .
\end{gathered}
$$

To see that $d$ has at least one zero for all values of $A, B$, in the suitable interval we study where the multiple zeroes of the curve are situated. For 
this purpose we compute a new discriminant, the one of $d$ with respect to $\lambda$, obtaining:

$$
s(A, B):=\operatorname{dis}\left(d_{A, B}(\lambda), \lambda\right)=-256 A B^{14}(B+1)\left(A^{2} B+2 A-1\right) \Delta(A, B)^{3} .
$$

Here $\Delta(A, B)$ is exactly the same function appearing in the proof of Theorem 4.3. Recall that this function does not vanish in the region of our interest, $A>0, B>0$ and $B \geq(1-2 A) / A^{2}$.

Therefore, the zeroes of $s(A, B)$ are located only on the Hopf bifurcation curve $A^{2} B+2 A-1=0$, and then for all the values of $A, B$ on this Hopf curve we can find a double zero of $d$, and consequently a $\lambda_{A, B}$ such that $S_{\lambda(A, B)}(x)$ does not change sign.

Moreover it is easy to see that, for instance for $A=4 / 5, B=1$, the function $d_{4 / 5,1}(\lambda)$ has two zeroes in the interval $(0, B /(1-A))$. If there exist some values of $A, B$ for which $d_{A, B}(\lambda)$ does not have a zero in this interval then, there must exist some other values of $A, B$ such that $d_{A, B}(\lambda)$ has a double root, and for these values $s(A, B)=0$. But the function $s(A, B)$ has no more zeroes, and consequently, for all values of $A, B>0, B \geq(1-2 A) / A^{2}$ there exists $\lambda_{A, B} \in(0, B /(1-A))$ such that $d_{A, B}\left(\lambda_{A, B}\right)=0$ and this $\lambda_{A, B}$ will be a double zero of $S_{\lambda(A, B)}(x)$, making this function not changing sign for $x \in[0,1]$. Hence the theorem follows.

4.3. Non-algebraicity of the limit cycle. We prove the following result which, by using Lemma 4.2, clearly implies the non-algebraicity of the limit cycle of system (1.1) when $A>0$ and $B>0$. This is a part of the statement of TheoremA.

Theorem 4.5. When $A(B+1) \neq 0$ the only invariant algebraic curves for system (4.2),

$$
\left\{\begin{array}{l}
\dot{x}=x(B x(1-x)-(B x+1) y) \\
\dot{y}=y(-A+x)
\end{array}\right.
$$

are the coordinate axes.

Proof. We use the method introduced in Subsection 2.3, with $y=\alpha(x) \equiv 0$, after interchanging $x$ and $y$. We write the algebraic curve

$$
F(x, y)=f_{0}(y)+f_{1}(y) x+\cdots+f_{k}(y) x^{k}=0,
$$

with $k \in \mathbb{N}$, and its corresponding cofactor, as

$$
K(x, y)=k_{0,0}+k_{0,1} y+k_{0,2} y^{2}+\left(k_{1,0}+k_{1,1} y\right) x+k_{2,0} x^{2},
$$

because the system is cubic. Then, by using equation (2.7), we obtain that $f_{0}(y)$ has to be a polynomial satisfying

$$
\left(k_{0,0}+k_{0,1} y+k_{0,2} y^{2}\right) f_{0}(y)+A y f_{0}^{\prime}(y)=0 .
$$

Since $A \neq 0$,

$$
f_{0}(y)=F_{0} y^{-\frac{k_{0,0}}{A}} \exp \left(-\frac{2 k_{0,1} y+k_{0,2} y^{2}}{2 A}\right), \quad F_{0} \in \mathbb{R} .
$$

Hence, the conditions for $f_{0}(y)$ to be a polynomial are

$$
k_{0,1}=k_{0,2}=0, \quad k_{0,0}=-m A, \quad m \in \mathbb{N}
$$


and then $f_{0}(y)=F_{0} y^{m}$. Studying the order one terms in $x$ in equation (2.6) we arrive to the new differential equation

$$
A y f_{1}^{\prime}(y)+(y-m A) f_{1}(y)=F_{0} y^{m}\left(m-k_{1,0}-k_{1,1} y\right) .
$$

Thus

$$
f_{1}(y)=-F_{0} k_{1,1} y^{m}+\frac{F_{0}\left(m-k_{1,0}\right)}{A} y^{m} e^{-\frac{y}{A}} \int_{1}^{y} \frac{e^{\frac{z}{A}}}{z} d z+K y^{m} e^{-\frac{y}{A}}, K \in \mathbb{R} .
$$

Hence the only polynomial solution happens when $K=0$ and $k_{1,0}=m$, giving that $f_{1}(y)=-F_{0} k_{1,1} y^{m}$.

Proceeding similarly to determine $f_{2}(y)$, the condition to be a polynomial is $k_{2,0}=k_{1,1} B$ and

$$
f_{2}(y)=\frac{F_{0} k_{1,1}\left(k_{1,1}+B\right)}{2} y^{m} .
$$

For the next step, the condition is $B(B+1)=0$, and then

$$
f_{3}(y)=-\frac{F_{0} k_{1,1}\left(k_{1,1}+B\right)\left(k_{1,1}+2 B\right)}{3 !} y^{m} .
$$

Hence the system can have algebraic solutions, different of the coordinate axes, only when either $B=0$ or $B=-1$. As the case $B=-1$ is not considered, it only remains to study what happens when $B=0$.

If $B=0$, by induction then we get

$$
F(x, y)=F_{0} y^{m}\left(\sum_{j=0}^{\infty} \frac{\left(-k_{1,1} x\right)^{j}}{j !}\right)=F_{0} y^{m} e^{-k_{1,1} x},
$$

which is never algebraic, but satisfies the corresponding equation (2.6). Indeed, in this case $F(x, y)$ is what it is usually called an exponential factor of the system.

Remark 4.6. In former theorem, as a consequence of the hypotheses of Theorem A, we have excluded the study of case $B=-1$. Nevertheless, in this case, it can be also proved that additionally we have $x=1$, as an invariant algebraic curve and that no more invariant algebraic curves exist.

Clearly, Theorem A follows by using all the results proved in this section.

\section{A Volterra System}

5.1. Deduction of the model. In mathematical ecology a family of natural models is given by the systems of the form

$$
\left\{\begin{array}{l}
\dot{x}=x(a(x)-b) y \\
\dot{y}=y(-c+d x)
\end{array}\right.
$$

where $x$ is the population density of the prey, $y$ is the population density of the predator and $a(x)$ describes the dynamics of multiplication of the prey in the absence of predators. The function considered in [3] is

$$
a(x)=\frac{a x(K-x)}{K(N+x)}
$$


where $0<N \ll K$. By rescaling the variables we obtain a dimensionless model of the form

$$
\left\{\begin{array}{l}
\dot{u}=\frac{u^{2}(1-u)}{n+u}-u v, \\
\dot{v}=\gamma v(u-m),
\end{array}\right.
$$

where all the parameters $\gamma, n, m$ are positive. Changing once more the time, since $n+u$ is positive in the first quadrant, the phase portrait of the above system coincides with the one of the polynomial system

$$
\left\{\begin{array}{l}
\dot{u}=u^{2}(1-u)-u v(n+u) \\
\dot{v}=\gamma v(u-m)(n+u) .
\end{array}\right.
$$

When $\gamma=1$ we get the system (1.2) that we are studying along this paper.

5.2. On the number of limit cycles. First, in next proposition we collect some results about local bifurcations and lower bounds for the number of limit cycles of system (1.2). After that, for proving Theorem B we will follow the same scheme that in the previous section.

Proposition 5.1. Consider system (1.2) in the first quadrant. Then:

(i) It does not have limit cycles when $m \geq 1$.

(ii) The curve $\left\{m=\sqrt{n^{2}+n}-n\right\}$ is an Andronov-Hopf bifurcation curve. More concretely:

- If $n<1 / 8$ an unstable limit cycle bifurcates from the origin for $m \gtrsim \sqrt{n^{2}+n}-n$

- At $(n, m)=(1 / 8,1 / 4)$ there is a codimension two weak focus and the family unfolds it,

- If $n>1 / 8$ a stable limit cycle bifurcates from the origin for $m \lesssim$ $\sqrt{n^{2}+n}-n$.

(iii) It has at least one limit cycle when $m<\sqrt{n^{2}+n}-n$.

(iv) It has at least two limit cycles when $n<1 / 8$ and $m \gtrsim \sqrt{n^{2}+n}-n$.

(v) For each $m<1 / 4$ there is some value $\widetilde{n}=\widetilde{n}(m)$, satisfying $\tilde{n}<$ $m^{2} /(1-2 m)$ for which the system has at least a semistable limit cycle.

Proof. (i) The critical points of system $(1.2)$ are $(0,0),(1,0)$ and $P=$ $(m, m(1-m) /(n+m))$. Moreover both axes are invariant by the flow. Since when $m \geq 1, P$ is not in the open first quadrant then it is clear that then the system does not have periodic orbits in this region.

(ii) The first Lyapunov quantity at the singular point $P$ is given by the divergence of the vector field at $P$,

$$
V_{1}=\frac{m\left(n(1-2 m)-m^{2}\right)}{m+n} .
$$

When $n=m^{2} /(1-2 m)$, next Lyapunov quantities at $P$, whenever it has sense and by using [6], are

$$
V_{3}=\frac{\pi}{4} \frac{(1-4 m)}{\sqrt{m}(1-m)^{2} \sqrt{1-2 m}}, \quad V_{5}=-\frac{64}{81} \pi \sqrt{2} .
$$

We note that at the point $(n, m)=(1 / 8,1 / 4)$ we have $V_{1}=V_{3}=0$ and $V_{5}<0$. Recall that the stability of the weak focus is given by the sign of the 
first non-null Lyapunov constant. If we choose $m=\frac{1}{4}-\delta, n=-\frac{m\left(m^{2}-\epsilon\right)}{2 m^{2}-m-\epsilon}$, then the Lyapunov constants are

$$
V_{1}=-\varepsilon<0, \quad V_{3}=\frac{64 \pi \delta}{\sqrt{1-4 \delta} \sqrt{2+8 \delta}(3+4 \delta)^{2}}>0, \quad V_{5}=-\frac{64}{81} \pi \sqrt{2}<0 .
$$

Consequently, choosing $0<\varepsilon \ll \delta \ll 1$, there are values of the parameters for which system (1.2) has, at least, two hyperbolic limit cycles, the inner one being unstable and the outer one being stable. In fact at the point $(n, m)=$ $(1 / 8,1 / 4)$ the system presents the complete unfolding of an Andronov-Hopf bifurcation of codimension two on the Hopf curve, see for instance [16]. The codimension one bifurcation appearing when $V_{1}=0, V_{3} \neq 0$, i.e. $n \neq 1 / 8$, can be similarly treated.

(iii)-(v) When $m<1$, the unstable separatix contained in the first quadrant of the saddle point at $(1,0)$ remains in the open triangle formed by the two coordinate axes and the straight line

$$
L:=\left\{(x, y): y=\frac{(n+1) m-(n+2)}{1+n}(x-1)\right\} .
$$

This can be easily seen by studying the vector field given by the system on $L$ and noticing that $(n+1) m-(n+2)<0$. Therefore its $\omega$-limit set has to be non-empty. When the point $P$ is unstable this limit set has to be a periodic orbit. This happens when $m<\sqrt{n^{2}+n}-n$ or when $m=\sqrt{n^{2}+n}-n$ and $n<1 / 8$. Therefore (iii) follows.

To prove (iv), notice that for $n<1 / 8$ and $m \gtrsim \sqrt{n^{2}+n}-n$ we have already proved in (ii) that a small limit cycle is born from $P$ and it is unstable. Therefore, the $\omega$-limit set of the separatrix of the saddle has to be a second periodic orbit surrounding the unstable one.

Finally to prove (v), fixed any $m=m^{*}$, with $0<m^{*}<1 / 4$, we consider the one-parameter family of vector fields $X_{n}$ associated to (1.2). Notice the following facts:

- By item (iv) we know that near the Andronov-Hopf curve, when $n \lesssim\left(m^{*}\right)^{2} /\left(1-2 m^{*}\right)$, the system $X_{n}$ has at least two limit cycles with opposite stabilities. For each $m^{*}$ we can take a value of $n$ with this property, say $n^{*}=n^{*}\left(m^{*}\right)$.

- When $n=0, X_{0}$ there are no limit cycles. This assertion follows because after removing the line of critical points $\{x=0\}$, it is a quadratic system with two invariant straight lines. It is well-known that these systems have no limit cycle. In fact this is also proved in Proposition 2.2 (ii).

- For $n \in\left[0, n^{*}\right]$, the Poincaré return map $\Pi_{n}$ is globally well defined on the open vertical segment $J_{n}$ joining the critical point $P$ and the $x$-axis. Moreover, by considering the displacement map $D_{n}:=$ $\Pi_{n}-\mathrm{Id}$, it can be seen that it does not have zeroes tending to the boundaries of $J_{n}$ when $n \in\left[0, n^{*}\right]$. Furthermore, for points near the boundaries of $J_{n}, D_{n}$ is always positive. This holds near $P$, because it is an attracting focus and near the other boundary of $J_{n}$ because, as we have already proved in (iii)-(iv), the $\omega$-limit set of the unstable 
separatrix of the saddle point, belonging to the first quadrant, also crosses $J_{n}$.

We can define $\widetilde{n}$ as follows:

$$
\widetilde{n}:=\inf \left\{n \in\left[0, n^{*}\right]: D_{n} \text { has some zero in } J_{n}\right\} .
$$

By its definition and the above properties, it holds that $\widetilde{n}>0, D_{\widetilde{n}} \geq 0$ and $D_{\widetilde{n}}$ has at least one zero in $J_{\widetilde{n}}$. Furthermore, since $D_{\widetilde{n}}$ is analytic and we know its behaviour at the boundaries of $J_{\tilde{n}}$, it has finitely many zeros in $J_{\widetilde{n}}$ (in fact exactly one if the conjecture of [3] is true). Clearly, each of the zeros of $D_{\widetilde{n}}$ corresponds to a semistable limit cycle of $X_{\widetilde{n}}$. Therefore item (v) follows.

Finally notice that although system (1.2) with $n>0$ and $m=0$ does not have periodic orbits in the first quadrant there are no critical points in the open first quadrant) we can not use the same type of reasoning to the family of vector fields which corresponds to fix $n$ and vary $m$. The reason is that when $m=0$ there is a change in the phase portrait of the system. As we have seen, this does not happen when $n=0$.

Theorem 5.2. Consider system (1.2) and assume that $m \in(0,1 / 2)$ and

$$
m<\min \left(\sqrt{n^{2}+n}-n, \frac{2 n}{1+3 n}\right)= \begin{cases}\frac{2 n}{1+3 n}, & n \leq \frac{1}{3}, \\ \sqrt{n^{2}+n}-n, & n \geq \frac{1}{3} .\end{cases}
$$

Then it has exactly one periodic orbit in the first quadrant and it is a stable limit cycle. The region of parameters for which the theorem applies is region $R_{1}$ in Figure 1.

Proof. By Proposition 5.1 we already know that under the hypotheses of the theorem the system has at least one limit cycle. To prove its uniqueness we will use again Theorem 2.3. For this case

$$
g(x)=x(1-x), \quad p(x)=x(n+x), \quad q(x)=(n-m) x+x^{2},
$$

$\gamma=m n$ and $K=1$. It is easy to see that our system satisfies all the hypotheses of the theorem, except perhaps the one concerning the sign of $\Phi$. Let us study this function. It writes as

$$
\Phi(x)=\frac{m x^{3}+n(3 m-2 n-2) x^{2}+m n(4 n+1) x-m n^{2}}{(n+x)^{3}(m-x)^{2}} .
$$

It suffices to study the sign of

$$
\Phi_{n, m}(x):=m x^{3}+n(3 m-2 n-2) x^{2}+m n(4 n+1) x-m n^{2}
$$

for $x \in[0,1]$ and $m$ and $n$ in the region considered. Notice that

$$
\Phi_{n, m}(0)=-n m^{2}<0 \quad \text { and } \quad \Phi_{n, m}(1)=(n+1)(3 m n-2 n+m)<0,
$$

where in this last inequality we have used that $n>m /(2-3 m)$. Moreover

$$
\operatorname{dis}\left(\Phi_{n, m}(x), x\right)=-4(n+1)^{2} m n^{3}\left(28 m n+m-8 n^{2}-8 n\right)\left(m^{2}+2 m n-n\right) .
$$

Since the factor $28 m n+m-8 n^{2}-8 n$ does not vanish in our region of parameters, arguing as in the proof of Theorem 4.3 we have proved that $\Phi_{n, m}(x) \leq 0$ for $x \in[0,1]$ and our theorem follows. 
Theorem 5.3. When $n>0$ and

$$
m \geq \max \left(\sqrt{n^{2}+n}-n, \frac{(2 n+1)^{2}}{4 n^{2}+8 n+5}\right)= \begin{cases}\frac{(2 n+1)^{2}}{4 n^{2}+8 n+5}, & n \leq \frac{\sqrt{2}-1}{2}, \\ \sqrt{n^{2}+n}-n, & n \geq \frac{\sqrt{2}-1}{2},\end{cases}
$$

system (1.2) does not have periodic orbits in the first quadrant. The region of parameters for which the theorem applies is region $R_{0}$ in Figure 1.

Proof. Recall that when $m \geq 1$ there are no critical points in the open first quadrant and so there are no periodic orbits. Therefore in the sequel we will assume $0<m<1$. We use again the criterion given in item (ii) of Theorem 2.1. For our system

$$
g_{0}(x)=x(1-x), g_{1}(x)=-n-x, h_{0}(x)=(x-m)(x+n), h_{1}(x) \equiv 0
$$

and $I=(0,1)$, because all the periodic orbits of the system have to be in the $\operatorname{strip}(0,1) \times(0,+\infty)$. Then

$$
S_{\lambda}(x):=(1-\lambda) x^{3}+(2 n(1-\lambda)+m \lambda) x^{2}+n((2 m-n) \lambda-1) x+m n^{2} \lambda
$$

and we need to find $\lambda$ such that $S_{\lambda}(x)$ does not change sign for $x \in[0,1]$.

Notice that

$$
S_{\lambda}(0)=n^{2} m \lambda, \quad \text { and } \quad S_{\lambda}(1)=(n+1)(\lambda(n+1)(m-1)+1) .
$$

Thus, we need that $\lambda(\lambda(m-1)(n+1)+1)>0$ and hence $\lambda$ has to be in the interval $I_{n, m}:=(0,1 /(n+1)(1-m))$. As in the proof of Theorem 4.4 we compute the following expression:

$$
\begin{aligned}
d_{n, m}(\lambda) & :=\operatorname{dis}\left(S_{\lambda}(x), x\right)= \\
& -4(n+1)\left[(m+n)^{3} n^{2} \lambda^{3}+c_{2} \lambda^{2}+c_{1} \lambda-n^{3}\right],
\end{aligned}
$$

where $c_{1}$ and $c_{2}$ are given polynomials depending on $n, m$. Then $d_{n, m}(0)>0$ and for $\bar{\lambda}=1 /((n+1)(1-m))$,

$$
d_{n, m}(\bar{\lambda})=\frac{n^{2}\left(\left(4 n^{2}+8 n+5\right) m-(2 n+1)^{2}\right)((3 n+1) m-2 n)^{2}}{(n+1)^{2}(-1+m)^{3}}
$$

Finally

$$
\begin{aligned}
s(n, m): & =\operatorname{dis}\left(d_{n, m}(\lambda), \lambda\right) \\
& =-16 m n^{9}(n+1)^{6}\left(m^{2}+2 m n-n\right)((28 n+1) m-8 n(n+1))^{3} .
\end{aligned}
$$

We claim that for the values $m$ and $n$ of the statement ${ }^{1}$ there exists $\lambda_{n, m}^{*} \in$ $I_{n, m}$ for which $d_{n, m}\left(\lambda_{n, m}^{*}\right)=0$. From the claim, we know that the cubic polynomial $S_{\lambda_{n, m}^{*}}(x)$ has a zero of multiplicity at least two and that $S_{\lambda_{n, m}^{*}}(0) S_{\lambda_{n, m}^{*}}(1)>0$. Then it is clear that $S_{\lambda_{n, m}^{*}}(x)$ does not change sign in $[0,1]$ and the theorem follows.

\footnotetext{
${ }^{1}$ For the values of $m$ and $n$ in the boundary of the region $R_{0}$, the value of $\lambda_{n, m}^{*}$ can also belong to the boundary of the interval $I_{n, m}$.
} 


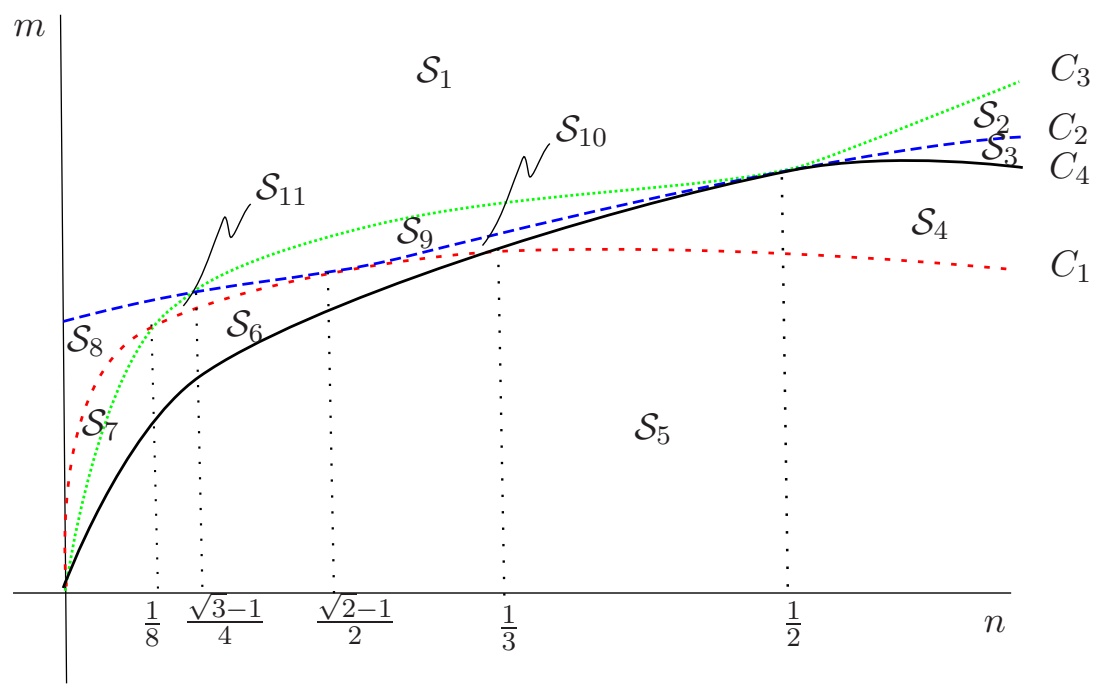

Figure 3. A sketch of the four algebraic curves $C_{1}, C_{2}, C_{3}, C_{4}$ and the connected components they define.

To end the proof it only remains to prove the above claim. To this end, consider the four algebraic curves:

$$
\begin{aligned}
& C_{1}(n, m)=m^{2}+2 n m-n=0, \quad(\text { the Hopf curve) } \\
& C_{2}(n, m)=\left(4 n^{2}+8 n+5\right) m-(2 n+1)^{2}=0, \\
& C_{3}(n, m)=(28 n+1) m-8 n(n+1)=0, \\
& C_{4}(n, m)=(3 n+1) m-2 n=0,
\end{aligned}
$$

and set $\mathcal{C}:=\cup_{i=1}^{4}\left\{(n, m): m>0, n>0, C_{i}(n, m)=0\right\}$. Define its complementary,

$$
\mathcal{S}:=\left\{(n, m) \in \mathbb{R}^{2},: m>0, n>0\right\} \backslash \mathcal{C} .
$$

Then, the number of zeroes of the function $d_{n, m}$ in the interval $I_{n, m}$ only depends on the connected component of $\mathcal{S}$ where the point $(n, m)$ lies. In other words, the change of the number of zeroes of $d_{n, m}$ in $I_{n, m}$ can only occur on $\mathcal{C}$. The values $(n, m) \in \mathcal{C}$, have to be treated separately. For the sake of brevity we omit the details for these cases.

A carefully study shows that the set $\mathcal{S}$ has eleven connected components. In Figure 3 it is drawn a sketch of the behavior of the four algebraic curves and of the components of $\mathcal{S}$. Comparing this figure with Figure 1 it is clear that the plot of the curves $C_{i}=0$ is not realistic. We only take into account their relative positions and intersections. We proceed in this way because the actual pictures determine very small regions which can not be seen in a realistic figure. For instance, the smallest one, $\mathcal{S}_{11}$, has triangular shape, its boundary has three smooth curves, one on each of the algebraic curves, 
and has three corners at:

$$
\begin{aligned}
& p_{1,2}:=\left(\frac{2-\sqrt{2}}{2}, \frac{\sqrt{2}-1}{2}\right) \simeq(0.293,0.207), \quad p_{1,2} \in C_{1} \cap C_{2}, \\
& p_{1,3}:=\left(\frac{1}{4}, \frac{1}{8}\right)=(0.25,0.125) \in C_{1} \cap C_{3}, \\
& p_{2,3}:=\left(\frac{7+2 \sqrt{3}}{37}, \frac{\sqrt{3}-1}{4}\right) \simeq(0.283,0.183), \quad p_{2,3} \in C_{2} \cap C_{3} .
\end{aligned}
$$

By picking one point on its interior, for instance $(n, m)=(9 / 50,281 / 1000)$, it is easy to prove that $d_{n, m}(\lambda)$ has no roots in $I_{n, m}$ and therefore our criterion does not work for all the values in $(n, m) \in \mathcal{S}_{11}$.

On the other hand, a big unbounded connected component is region $\mathcal{S}_{1}$ in the above-mentioned figure,

$$
\mathcal{S}_{1}:=\left\{(n, m): n>0, m>\max \left(\frac{(2 n+1)^{2}}{4 n^{2}+8 n+5}, \frac{8 n(n+1)}{28 n+1}\right)\right\} .
$$

A point in its interior is $(n, m)=(1 / 2,1 / 2)$ and $I_{1 / 2,1 / 2}=(0,4 / 3)$. Then

$$
d_{1 / 2,1 / 2}(\lambda)=\frac{1}{64}\left(-96 \lambda^{3}+261 \lambda^{2}-216 \lambda+48\right)
$$

and this polynomial has a real root $\lambda^{*} \simeq 0.3538 \in I_{1 / 2,1 / 2}$. Hence for $m=$ $n=1 / 2$, the function $S_{\lambda^{*}}(x)$ is positive or zero in $[0,1]$. This fact implies that we have proved the non-existence of periodic orbits for all the values $(n, m) \in \mathcal{S}_{1}$.

We do a similar study for the remaining nine regions. In short, we have proved that only in six of them, $\mathcal{S}_{1}, \mathcal{S}_{2}, \mathcal{S}_{3}, \mathcal{S}_{4}, \mathcal{S}_{9}$ and $\mathcal{S}_{10}$, the function $d_{n, m}$ has at least one zero in the interior of $I_{n, m}$. The union of these regions, together with the corresponding boundaries between them, form the region of parameters given in the statement. Hence the claim is proved.

\subsection{Non-algebraicity of the limit cycles.}

Theorem 5.4. When $n(n+1) m \neq 0$, the only invariant algebraic curves for system (1.2),

$$
\left\{\begin{array}{l}
\dot{x}=x(x(1-x)-(n+x) y) \\
\dot{y}=y\left(-m n+(n-m) x+x^{2}\right),
\end{array}\right.
$$

are the coordinate axes.

Sketch of the proof. Following the same notation and steps as in the proof of Theorem 4.5 we obtain that

$$
f_{0}(y)=F_{0} y^{-\frac{k_{0,0}}{n m}} \exp \left(-\frac{2 k_{0,1} y+k_{0,2} y^{2}}{2 n m}\right), \quad F_{0} \in \mathbb{R} .
$$

Former expression is polynomial when

$$
k_{0,1}=k_{0,2}=0, \quad k_{0,0}=-n m p, \quad p \in \mathbb{N} .
$$

By continuing the same procedure, until third order terms in $x$, we obtain the following set of necessary conditions to have an invariant algebraic curve:

$$
k_{1,0}=(n-m) p, \quad k_{2,0}=\frac{n p-k_{1,1}}{n}, \quad n+1=0 .
$$

Hence, since we assume that $n+1 \neq 0$, the proof finishes. 
Remark 5.5. For system (1.2) we note that if $n=-1$ then, besides the own axes, the unique invariant algebraic curve is the straight line $x=1$. In this case, we can proceed with the method given in the previous proof obtaining that the invariant curve writes as

$$
F(x, y)=F_{0} y^{p} \sum_{j=0}^{\infty}\left(\begin{array}{c}
k_{1,1}+j-1 \\
j
\end{array}\right) x^{j}=0 .
$$

This curve is algebraic only when $-k_{1,1}=q \in \mathbb{N}$ and, in this case, it is $F(x, y)=F_{0} y^{p}(1-x)^{q}$.

Theorem B is a direct consequence of all the results proved in this section.

\section{REFERENCES}

[1] A.A. Andronov, E.A. Leontovich, I.I. Gordon, A.G. Meyer, Qualitative theory of second-order dynamic systems. John Wiley \& Sons, New York-Toronto, Israel Program for Scientific Translations, Jerusalem-London, 1973.

[2] A.A. Andronov, E.A. Leontovich, I.I. Gordon, A.G. Meyer, Theory of bifurcations of dynamics systems on a plane. John Wiley \& Sons, New York-Toronto, Israel Program for Scientific Translations, Jerusalem-London, 1973.

[3] A. D. Bazykin, A. I. KhiBnik, Biophysics of complex systems. Rigid regime of excitation of auto-oscillations in a model of the Volterra type, Biophysics 5 (1981), 866-869.

[4] H. I. Freedman, Deterministic mathematical models in population ecology. Monographs and Textbooks in Pure and Applied Mathematics, 57. Marcel Dekker, Inc., New York, 1980.

[5] A. Gasull, H. Giacomini, J. Torregrosa, Explicit non-algebraic limit cycles for polynomial systems. J. Comput. Appl. Math. 200 (2007), 448-457.

[6] A. Gasull, J. Torregrosa, A new approach to the computation of the Lyapunov constants. The geometry of differential equations and dynamical systems. Comput. Appl. Math. 20 (2001), 149-177.

[7] H. Hirashita, Cyclic changes in dust-to-gas ratio, The Astrophysical Journal 531 (2000), 693-700.

[8] H. Hirashita, H. Kamaya, Application of the limit-cycle model to star formation histories in spiral galaxies: variation among mophological types, The Astrophysical Journal 120 (2000), 728-732.

[9] S.B. Hsu, T.W. HuAng, Global stability for a class of predator-prey systems $\mathbf{5 5}$ (1995), 763-783.

[10] X. Huang, Y. Wang, A. Cheng, Limit cycles in a cubic predator-prey differential system, J. Korean Math. Soc. 43 (2006), 829-843.

[11] S. Ikeuchi, H. Tomita, Cyclic phase changes of interstellar medium, Publ. Astron. Soc. Japan 35 (1983), 77-86.

[12] H. Kamaya, Mass-loss, episodic star formation and the HI envelope of blue compact dwarf, Astronomy \& Astophysics, 434 (2005), 163-165.

[13] H. Kamaya, T.T Takeuchi, A Nonlinear-Open-System Model for Star Formation in Spiral Galaxies, Publ. Astron. Soc. Japan 49 (1997), 271-273.

[14] Y. Kunng, Global stability of Gause-type predator-prey systems J. Math. Biol. 28 (1990), 463-474.

[15] Y. Kuang, H. I. Freedman, Uniqueness of limit cycles in Gause-type models of predator-prey systems. Math. Biosci. 88 (1988), 67-84.

[16] Y. A. Kuznetsov, Elements of applied bifurcation theory. Applied Mathematical Sciences. 112. New York, Springer, 1998.

[17] H.N. Moreira, On Liénard's equation and the uniqueness of limit cycles in predatorprey systems, J. Math. Biol. 28 (1990), 341-354.

[18] M. L. Rosenzweig; R. H. MCARThuR, Graphical representation and stability conditions of predator-prey interactions, Am. Nat. 47 (1963), 209-223. 
Dept. De Matemàtiques i Informàtica, Universitat de les Illes Balears, 07122 Palma de Mallorca, Illes Balears. Spain

E-mail address: chus.alvarez@uib.es

Dept. De Matemàtiques, Universitat Autònoma de Barcelona, Edifici C 08193 Bellaterra, Barcelona. Spain

E-mail address: gasull@mat.uab.cat

Dept. De Matemàtiques i Informàtica, Universitat de les Illes Balears, 07122 Palma de Mallorca, Illes Balears. Spain

E-mail address: rafel.prohens@uib.cat 